\title{
3 Research Square

\section{Altered Expression of IL-18, IL-18 Binding Protein and IL-18 Receptor in Blood Monocytes of Patients with Allergic Rhinitis}

Junling Wang

China Medical University

Fangqiu Gu

China Medical University

Zhi Li

First Affiliated Hospital of Jinzhou Medical University

Mengmeng Zhan

Shenyang Medical College

Ling Wang

Shenyang Medical College

Huiyun Zhang

Shenyang Medical College

\section{Dong Chen}

First Affiliated Hospital of Jinzhou Medical University

Hua Xie

General Hospital of Northern Military Area

\section{Ruonan Chai}

General Hospital of Northern Military Area

Nan Zhao

First Affiliated Hospital of Jinzhou Medical University

\section{Ruiming Yang}

First Affiliated Hospital of Jinzhou Medical University

\section{Yalin Hu}

First Affiliated Hospital of Jinzhou Medical University

Shaoheng He ( $\square$ shoahenghe@126.com )

College of Basic Medical Sciences, China Medical University https://orcid.org/0000-0002-4230-7639

\section{Research article}

Keywords: IL-18, IL-18 receptor, IL-18 binding protein, Monocyte, Allergen, Allergic rhinitis 
Posted Date: November 11th, 2020

DOl: https://doi.org/10.21203/rs.3.rs-67682/v1

License: (c) (i) This work is licensed under a Creative Commons Attribution 4.0 International License. Read Full License 


\section{Abstract}

Background: Interleukin (IL)-18 is emerging as an attractive participant in allergic rhinitis (AR). However, correlation of IL-18 with IL-18 binding protein (BP) in plasma, and expression of IL-18, IL-18BP and IL-18 receptor $(R)$ in AR blood monocytes remain obscure. We therefore investigated IL-18, IL-18BP and IL-18R expression in monocytes using flow cytometric analysis, murine AR model, and quantitative real-time PCR in the present study.

Results: Plasma IL-18 and IL-1 $\beta$ in AR patients was higher than those in healthy control subjects. Free (f) IL-18 had a high correlation with IL-18BP, IL-1 $\beta$ and TNF- $\alpha$ in AR plasma. Proportion of IL-18 ${ }^{+}$ monocytes was increased, whereas IL-18BP ${ }^{+}$monocytes were decreased in blood of patients with AR. It was found that Platanus pollen allergen extract provoked the elevated expression of IL-18 and IL-18R in AR blood monocytes. Dermatophagoides pteronyssinus, Artemisia sieversiana wild and Platanus pollen allergen extracts enhanced IL-18R protein and mRNA expression in isolated primary monocytes from AR patients. Moreover, numbers of macrophages and IL-18R macrophages in nasal lavage fluid (NLF) were increased, and levels of IL-18 in both plasma and NLF were elevated in AR mice.

Conclusions: These suggest that IL-18 is likely to participate in the development of AR as a causative factor; therefore, it could be a therapeutic target for AR.

\section{Background}

IL-18, initially discovered as interferon (IFN)-y-inducing factor constitutively expressed by monocytes and macrophages, and plays regulatory roles in both innate and adaptive immunity. It is reported that polymorphisms of IL-18 gene was associated with AR [1-5], and up-regulated IL-18 is found in both nasal secretion and serum of AR patients [6,7]. It is also observed that elevated serum IL-18 during natural pollen exposure is closely associated with bronchial hyperresponsiveness in seasonal AR (SAR) patients [8]. These implicate that monocyte- and macrophage-derived IL-18 likely contributes to the pathogenesis of AR.

IL-18 binds initially to IL-18R then initiates MyD88-dependent signal pathway and exert immunomodulatory functions [9]. IL-18 binding protein (IL-18BP) is an endogenous soluble antagonist that specifically inhibits IL-18 action by binding to IL-18 with high affinity [10]. Free serum IL-18BP is present at 20fold higher levels than free IL-18 in physiological status [11]; however, under allergic conditions IL-18 may be in excess [12]. These suggest an imbalance between IL-18 and IL-18BP expression may account for increased IL-18 activity in AR. Indeed, we reported recently that the role of IL18 in atopic asthma is determined by the balance of IL-18/IL-18BP/IL-18R [13]. Since level of circulating IL-18BP in AR plasma/serum has not been reported, we examined level of IL-18BP in AR plasma in the present study.

It has been reported that pollen allergen specific subcutaneous immunotherapy induced increased serum IL-18 in AR patients [14], pollen allergen extract could provoke IL-18 mRNA expression in PBMCs of 
patients with AR undergoing allergen immunotherapy [15-17], suggesting that allergens may contribute to AR through PBMCs and IL-18-related mechanisms. Since monocytes are one major cell type of PBMCs, and little is known about expression of IL-18, IL-18BP and IL-18R at protein and mRNA levels in monocytes of $A R$, particularly upon allergen challenge. Therefore, the aim of the present study is to investigate levels of IL-18 and IL-18BP in AR plasma, expression of IL-18, IL-18BP and IL-18R in monocytes of patients with $A R$, and influence of allergens on their expression.

\section{Results}

\section{Elevated level of IL-18 in plasma of patients with pAR and sAR}

Using ELISA kits, it was observed that levels of tIL-18 (Fig. 1A) and fIL-18 (Fig. 1C) in plasma of patients with $\mathrm{pAR}$ and SAR were elevated in comparison with $\mathrm{HC}$. In contrast, there were no significant differences of plasma levels of tIL-18BP (Fig. 1B) and fIL-18BP (Fig. 1D) between patients with pAR or sAR and HC being observed. It was found that the molar concentration ratio of fIL-18BP/fIL-18 for HC (16.5) was markedly greater than that for patients with SAR (9.7) (Fig. 1E), indicating that IL-18 is likely to play a role in SAR. Moreover, significant correlations between fIL-18 and fIL-18BP were observed in patients with pAR, SAR and HC (Fig. 1F).

\section{Elevated levels of IL-1 $\beta$ and TNF- $\alpha$ in plasma of patients with SAR}

Since monocytes and macrophages are involved in the pathogenesis of AR possibly by overproducing IL$1 \beta$ and TNF- $\alpha$ [18-21], we examined levels of IL-1 $\beta$ and TNF- $\alpha$ in plasma of patients with PAR, sAR and $\mathrm{HC}$ using human bio-plex panel kit. As shown in Fig. 2A, plasma level of IL-1 $\beta$ was generally low, nevertheless it appeared that plasma level of IL-1 $\beta$ in pAR and SAR patients were higher than that in HC. The plasma level of TNF- $a$ in sAR patient $(8.7 \mathrm{pg} / \mathrm{mL})$, but not the patients with pAR was significantly higher than that in $\mathrm{HC}(7.2 \mathrm{pg} / \mathrm{mL})$ (Fig. 2B). Moreover, fIL-18, IL-1 $\beta$ and TNF- $\alpha$ were shown to be correlated well between each other in plasma of patients with pAR and SAR (Fig. 2C).

Increased expression of IL-18 and IL-18R, and decreased expression of IL-18BP in monocytes of patients with PAR and sAR

Since fIL-18, IL-1 $\beta$ and TNF- $\alpha$ were correlated well between each other in plasma of patients with pAR and SAR, and IIL-18 and fIL-18 in plasma of patients with PAR and SAR were elevated in comparison with HC, we investigated the expressions of IL-18, its specific receptor IL-18R, and its natural specific neutralizer IL$18 \mathrm{BP}$ in peripheral blood monocytes in the present study. The results showed that the proportion of IL-18 ${ }^{+}$ monocytes was increased by 4.2 and 9.1 fold, and IL-18BP ${ }^{+}$monocytes was decreased by $77.5 \%$ and $56.0 \%$ in PAR and sAR patients, respectively when compared with HC (Fig. 3B, C). PPAE seemed to upregulate IL-18 and IL-18R expression in monocytes of pAR and SAR patients, respectively (Fig. 3B, C). 
As for the expression intensity of a single positive cell (mean fluorescence intensity, MFI), pAR and sAR patients appeared to have lower MFI of IL-18BP in monocytes than that in HC (Fig. 3D ii, E), and SAR patients had higher MFI of IL-18R on monocytes than that in HC and pAR patients (Fig. 3D iii, E).

Moreover, all allergens tested in this study including ASWAE, PPAE and DPAE enhanced the MFI of IL-18 ${ }^{+}$ monocyte in HC, and ASWAE increased the MFI of IL-18 ${ }^{+}$monocyte in SAR patients (Fig. 3D i, E).

\section{Allergens and IFNy induced alteration of IL-18, IL-18BP and IL-18R expression in isolated monocytes}

In order to evaluate the direct effects of allergens on the expression of IL-18, IL-18BP and IL-18R in purified monocytes (purity up to $99.7 \%$ ), we co-cultured DPAE, ASWAE, PPAE or IFNy with purified monocytes in 12-well cell culture plate, and examined expression of IL-18, IL-18BP and IL-18R by flow cytometry. The results showed that proportion of IL-18BP monocytes in AR patients was decreased compared with $\mathrm{HC}$ (Fig. 4A, C), and IL-18BP expression appeared to be up-regulated in HC following the stimulation of DPAE for 10 min (Fig. 4A, C). It was also shown that ASWAE, PPAE and IFNy induced the elevated expression of IL-18R in monocytes of AR patients at $10 \mathrm{~min}$ following incubation. Moreover, DPAE increased the expression of IL-18R in AR patients at 30 min following stimulation (Fig. 4A, D). However, number of IL-18 $8^{+}$monocytes in AR patients was decreased compared with that in HC (Fig. 4A, B), which was in contrast with the result seen in Fig. 3B and 3C.

In terms of MFI, DPAE and IFNy seemed to down-regulate MFI of IL-18BP ${ }^{+}$monocyte in AR patients at 60 min following incubation (Fig. 4E i, G). While the enhanced MFI of IL-18R ${ }^{+}$monocyte in patients with AR was observed in comparison with HC (Fig. 4E ii, H), DPAE increased the MFI of IL-18R in HC at 10 min following incubation (Fig. 4E ii, H). However, allergens and IFNy tested in the present study had little effect on IL-18 expression in monocytes (Fig. 4A, B, F).

\section{Allergen Induced II-18r Mrna Expression In Primary Monocytes}

In order to further understand the effects of allergens on IL-18, IL-18BP and IL-18R expression in monocytes, we examined expression of IL-18, IL-18BP and IL-18R mRNAs in primary monocytes using qPCR technique. As seen in Fig. 5, ASWAE, PPAE, DPAE upregulated expression of IL-18R mRNA in monocytes of AR patients by 2.5, 2.5 and 4.6 fold, respectively at 30 min following challenge. At 60 min following challenge, only DPAE-induced expression of IL-18R mRNA in monocytes of AR patients was observed. ASWAE-provoked expression of IL-18R mRNA in monocytes was also found in HC at 30 min following challenge. Allergens tested had little effect on expression of IL-18 and IL-18BP mRNAs in monocytes of $\mathrm{AR}$ and $\mathrm{HC}$ following 10, 30 and 60 min challenge periods (data not shown).

Increased level of IL-18 in both plasma and NLF of AR mice 
To understand further the role of IL-18 in AR, influence of OVA challenge on IL-18 and IL-18BP production in AR mice was examined. The results showed that IL-18 (Fig. 6A, B), but not IL-18BP (data not shown) levels were elevated in both plasma and NLF of AR mice.

\section{Down-regulated expression of IL-18R in blood monocytes and up-regulated expression of IL-18R in NLF macrophgaes of AR mice}

To confirm the role of IL-18R in AR, we examined IL-18R expression in both blood monocytes and NLF macrophages of AR mice. Compared with HM group, while the number of monocytes (Fig. 7A, B) and MFI of IL-18R ${ }^{+}$monocyte (data not shown) in blood leukocytes had little change, the percentage of IL-18R ${ }^{+}$ monocytes was reduced by $58.3 \%$ in AR mice (Fig. $7 A, C$ ). In contrast, the numbers of $F 4 / 80^{+}$ macrophages and IL-18R ${ }^{+}$macrophages were increased approximately 1.2 (Fig. 7D, E) and 1.4 fold (Fig. 7D, F), respectively in AR mice.

\section{Discussion}

IL-18 is a pro-inflammatory cytokine that induces IFN-y production, which is closely related to the pathophysiologic mechanism of allergic respiratory disorders [22]. In the present study, we showed that free plasma IL-18 was elevated in PAR and sAR patients, sAR patients had a decreased IL-18BP/IL-18 ratio (9.7), and free IL-18 correlated well with free IL-18BP in the plasma of both pAR patients and SAR patients, indicating that the imbalance between IL-18 and IL-18BP is likely to be crucial to the development of AR as a molar excess of 10 of IL-18BP over IL-18 is required to decrease a pathological level of $400 \mathrm{pg} / \mathrm{ml}$ of IL-18 to a level of a $\mathrm{HC}(40 \mathrm{pg} / \mathrm{ml})$ [11]. Since IL-18BP has neutralizing capacity of IL-18 [10] and excessive free IL-18 can cause inflammatory conditions [12, 23], our data suggest that IL18 may participate in the development of AR as a causative factor.

In the present study, we also observed that elevated levels fIL-18, IL-1 $\beta$ and TNF-a were correlated well between each other in plasma of patients with pAR and sAR. Given the fact that IL-1 $\beta$ and TNF- $\alpha$ are mainly produced by monocytes and macrophages [24, 25], we anticipate that the elevated levels of fIL-18, IL-1 $\beta$ and TNF- $\alpha$ are at least partially originated from monocytes. Our observation that the proportion of $\mathrm{IL}-18^{+}$monocytes was increased in PAR and SAR patients may support the above anticipation, we hence believe that monocyte-derived IL-18 is likely to play a role in AR. The decreased IL-18BP ${ }^{+}$monocytes were found in AR peripheral blood may help to understand excessive fIL-18 in AR plasma as reduced IL-18BP production can eliminate IL-18/IL-18BP complex, and consequently free more IL-18 in the plasma. The enhanced MFI of IL-18R on monocytes of SAR patients suggest that IL-18 may act on monocytes through its receptor, which could implicate a paracrine mechanism that monocytes secrete IL-18, and IL-18 act on adjacent monocytes via IL-18R.

The results in the present study that PPAE upregulates IL-18 and IL-18R expression in monocytes of pAR and SAR patients, and ASWAE enhances the MFI of IL-18 $8^{+}$monocyte in SAR patients suggest that airborne allergens can directly affect IL-18 and IL-18R expression in monocytes even though direct 
contact of allergens with blood monocytes hardly occurs in the body. Unexpectedly, the MFI of IL-18 monocyte in $\mathrm{HC}$ can be enhanced by all allergens tested in this study including ASWAE, PPAE and DPAE. Since IL-18 plays regulatory roles in both innate and adaptive immunity [26], elevated serum IL-18 during natural pollen exposure is closely associated with bronchial hyperresponsiveness in seasonal AR patients [8], and monocytes are one major source of IL-18, the enhanced expression of IL-18 on monocytes may help to promote sensitization of $\mathrm{HC}$ to airborne allergens. The finding that synergy of IL-5 and IL-18 in eosinophil mediated pathogenesis of allergic diseases [27] may also support the view that IL-18 promote allergy.

Using primary monocytes, the elevated expression of IL-18R in monocytes of AR patients induced by allergens ASWAE, PPAE and DPAE was confirmed at both protein and mRNA levels, suggesting that allergen-induced upregulation of expression of IL-18R is most likely a direct event. Although the proportion of IL-18BP ${ }^{+}$monocytes in AR patients was decreased compared with $\mathrm{HC}$, and DPAE seemed to down-regulate MFI of IL-18BP ${ }^{+}$monocyte in AR patients, allergens tested had little effect on expression of IL-18BP mRNAs in monocytes of AR and HC, suggesting that reduced IL-18BP expression in monocytes most likely occurred at protein synthesis process such as elongation, transport or modification stages.

On the other hand, compared with $\mathrm{HC}$ blood, less proportion of $\mathrm{IL}-18^{+}$populations were found in primary monocytes from peripheral blood of AR patients. This is an unexpected result considering our previous observation that the proportion of IL-18 $18^{+}$monocytes was increased in peripheral blood of pAR and SAR patients. It is difficult to explain these conflict results without performing more detailed investigation, but the isolation procedure and individual difference between patients may take into account.

The results that IL-18 levels were elevated in both plasma and NLF of AR mice following OVA challenge support our observation that IL-18 level was increased in patients with AR. Since expression of IL-18R in blood monocytes appeared to be down-regulated and expression of IL-18R in NLF macrophages was upregulated in AR mice, increased IL-18 may contribute to AR through macrophages or IL-18R expressing cells other than monocytes.

\section{Conclusion}

In summary, we demonstrated for the first time that enhanced fIL-18 in AR plasma, and upregulated IL-18 and IL-18R expression in monocytes of AR patients, which implicate strongly that IL-18 may serve as a causative factor for AR. Regulation of expression of IL-18, IL-18BP and IL-18R in monocytes by specific allergens suggests allergens can directly act on monocytes, thereafter modify IL-18, IL-18BP and IL-18R expression. These observations imply that monocyte-derived IL-18 is likely to contribute to the pathogenesis of AR, and therefore IL-18 could be therapeutic target for AR.

\section{Methods}

\section{Reagents}


The following reagents were purchased from Biolegend (San Diego, CA, USA): human RBC lysis buffer, Brilliant Violet 510 ${ }^{\text {TM }}$ (BV510)-conjugated donkey anti-rabbit IgG polyclonal antibody, PE/Cy7-conjugated mouse anti-human CD14 monoclonal antibody, BV510-conjugated rat anti-mouse Gr-1 monoclonal antibody, BV510-conjugated rat anti-mouse F4/80 monoclonal antibody, PerCP-conjugated rat antimouse CD11b monoclonal antibody, human Fc receptor blocking solution, rat anti-mouse CD16/32 antibody, Brefeldin A, Zombie NIR ${ }^{\text {TM }}$ Fixable Viability Kit, and Zombie Green ${ }^{\text {TM }}$ Fixable Viability Kit. Mouse IL-18BPd DuoSet ELISA kit, APC-conjugated mouse anti-human IL-18Ra monoclonal antibody, PEconjugated mouse anti-human IL-18 monoclonal antibody, APC-conjugated rat anti-mouse IL-18Ra monoclonal antibody and their respective isotype controls (catalog \#: IC002A, IC002P, IC005A) were supplied by R\&D Systems (Minneapolis, MN, USA). Rabbit anti-human IL-18BPa and rabbit IgG isotype control were obtained from Novus Biologicals (Minneapolis, MN, USA). IFNy was supplied by PeproTech (Rocky Hill, NJ, USA). Trypan blue dye and Ovalbumin (OVA, Grade V) were purchased from Sigma-Aldrich (St. Louis, MO, USA). Human IL-18BPa and IL-18 ELISA kits were from ImmunoWay Biotechnology Company (Plano, TX, USA) and ExCell Bio (Shanghai, China), respectively. Cytofix/Cytoperm ${ }^{\text {TM }}$ Fixation/Permeabilization Solution Kit was bought from BD Biosciences (Beldford, MA, USA). Fetal bovine serum (FBS, Hyclone), penicillin-streptomycin antibiotic mixture and RPMI 1640 medium were obtained from Gibco BRL (Grand Island, NY, USA). Alhydrogel ${ }^{\circledR}$ adjuvant was bought from InvivoGen (San Diego, CA, USA). RBC Lysis Buffer (Multi-species), mouse IL-18 ELISA kit and TRIzol reagent were purchased from Invitrogen (Carlsbad, CA, USA). anti-human CD14 MicroBeads and autoMACS Running Buffer-MACS Separation Buffer, and Lymphoprep ${ }^{\text {TM }}$ were obtained from Miltenyi Biotec (Bergisch Gladbach, Germany) and AXIS-SHIELD PoC AS (N-0504 Oslo, Norway), respectively. Dermatophagoides pteronyssinus allergen extract (DPAE) was bought from Greer Laboratories, Inc. (Lenoir, NC, USA). Artemisia sieversiana wild allergen extract (ASWAE) and Platanus pollen allergen extract (PPAE) were purchased from Macro Union Pharmaceutical Co. Ltd (Beijing, China). Allergens for skin prick tests were supplied by ALK-Abelló, Inc. (Denmark). Most of the general-purpose chemicals such as salts and buffer components were of analytical grade.

\section{Subjects And Animals}

General characteristics of 33 patients with perennial allergic rhinitis (pAR), 9 patients with sAR and 25 healthy control subjects $(\mathrm{HC})$ recruited in this study were summarized in Supplement table 1. The diagnosing criteria of pAR and sAR were conformed to the Chinese Society of Allergy Guidelines for Diagnosis and Treatment of Allergic Rhinitis [28].

The experiment was approved by the ethical committees of the First Affiliated Hospital of Jinzhou Medical University and the General Hospital of Northern Military Area. $5 \mathrm{~mL}$ of peripheral blood samples were drawn into $\mathrm{K}_{2}$ EDTA containing tubes after obtaining written informed consent from each participant, and centrifuged at $450 \times g$ for $10 \mathrm{~min}$. The cells and plasma were collected separately for flow cytometric and ELISA analysis. For CD $14^{+}$monocytes isolation study, approximately $180 \mathrm{~mL}$ of peripheral blood was taken from each donor. 
Five-week-old female BALB/c mice, weighting 18-22 g, were purchased from Vital River Laboratory Animal Technology Co., Ltd. (Beijing, China), and maintained in specific pathogen-free facilities with free access to standard rodent chow and water, at a constant temperature $23-28^{\circ} \mathrm{C}$ and relative humidity of $60-75 \%$ of the First Affiliated Hospital of Jinzhou Medical University maintained as described previously [12]. The animal experiment procedures were authorized by the Animal Care Committee at Jinzhou Medical University.

\section{Isolation Of Cd14 Monocytes And Allergen Challenge Test}

$\mathrm{CD}_{14}{ }^{+}$cells were enriched by density gradient centrifugation and positive selection on magnetic cell sorting (MACS) according to the manufacturer's instructions. Purities of recovered CD14 ${ }^{+}$cells were evaluated by flow cytometry with PE/Cy7-conjugated anti-human CD14 antibody.

To further investigate the direct action of allergen on the expression of IL-18, IL-18BP and IL-18R in monocytes, the isolated monocytes at a density of $1 \times 10^{6}$ per mL were cultured in RPMI 1640 medium containing $3 \% \mathrm{FBS}$ and $100 \mathrm{U} / \mathrm{ml}$ penicillin/streptomycin in a 12-well cell culture plate (Nest, Wuxi, China) in the presence or absence of ASWAE, PPAE, DPAE (all at a concentration of $1.0 \mu \mathrm{g} / \mathrm{mL}$ ) or IFNy (as positive control) at $5 \mathrm{ng} / \mathrm{mL}$ for 10,30 and 60 min, respectively at $37^{\circ} \mathrm{C}$ in a $5 \%(\mathrm{v} / \mathrm{v}) \mathrm{CO}_{2}$, watersaturated atmosphere. Brefeldin A at $2 \mu \mathrm{g} / \mathrm{mL}$ was added in wells for detecting the intracellular expression of IL-18 and IL-18BP before stimulation. Cells were then harvested and centrifuged at $450 \times g$ for 10 min at $4^{\circ} \mathrm{C}$. Cell pellets containing approximately $0.5 \times 10^{6}$ and $1 \times 10^{6}$ cells were resuspended in PBS for flow cytometric analysis and in TRIzol reagent for RT-PCR, respectively.

Flow cytometric analysis of IL-18, IL-18BP and IL-18R in human peripheral blood monocytes and isolated CD14 ${ }^{+}$monocytes

The procedures for detecting IL-18, IL-18BP and IL-18R expression in human peripheral blood monocytes were mainly adopted from a previous study by Zhang et al [13]. Briefly, whole blood cells were challenged with or without ASWAE, PPAE, or DPAE (all at a concentration of $1.0 \mu \mathrm{g} / \mathrm{mL}$ ) for $1 \mathrm{~h}$.

For cell surface molecules, whole blood cells were preincubated with human Fc receptor blocking solution and Zombie Green dye [29], then stained with PE/Cy7 conjugated anti-human CD14 and APC conjugated anti-human IL-18Ra antibodies. Following erythrocyte lysis, leukocytes were analyzed with FACSVerse flow cytometer (BD Biosciences, San Jose, CA, USA). An irrelevant isotype- and concentration-matched antibody of anti-human IL-18Ra was used for fluorescence minus one (FMO) control. Dead cells and doublets were discriminated by SSC-A-live/dead cell dye and FSC-H-FSC-A gating strategies. As for isolated monocytes, cells were processed as above.

For intracellular molecules, resultant leukocytes prepared as above were fixed and permeabilized, and stained with anti-human IL-18BP and PE conjugated anti-human IL-18 antibodies, which was followed by 
the addition of BV510-conjugated donkey anti-rabbit polyclonal antibody. These procedures were applicable to isolated monocytes.

\section{Establishment Of Mouse Ar Model}

A total of 14 mice were randomly divided into control and AR groups. OVA-induced AR mouse model was mainly adopted from a previous study by Mo JH et al [30]. Briefly, mice were sensitized on days 0,7 and 14 by intraperitoneal injection of $25 \mu \mathrm{g}$ OVA emulsified in $1 \mathrm{mg}$ of alhydrogel. On days 21-27 mice were challenged by intranasal instillation with $500 \mu \mathrm{g}$ of OVA dissolved in PBS (10 $\mu \mathrm{L} /$ nostril) once daily. For control experiments, control mice received vehicle only instead of OVA solution. At $24 \mathrm{~h}$ following the last OVA challenge, mice were sacrificed by eyeball enucleation, blood and nasal lavage fluid (NLF) samples were thereafter collected. Total cells were determined and centrifuged as above, amongst cells were used for flow cytometric analysis, plasma and NLF supernatant was aliquoted and frozen at $-80^{\circ} \mathrm{C}$ for ELISA assay.

To evaluate allergic symptoms, numbers of sneezing and nasal-rubbing motions of all survived mice during the first 15 min after each challenge were recorded, and compared with healthy control mice (HM) by observers blinded to the study. As presented in Supplement Fig. 1A and Fig. 1B, the numbers of nasal rubbing and sneezing motion in AR mice $(n=7)$ were substantially higher than that in $\mathrm{HM}(n=7)$ during a 7-day observation period.

Flow cytometric analysis of IL-18R expression in mouse blood monocytes and NLF macrophages

To detect IL-18R expression in mouse blood monocytes, whole blood cells were preincubated with antimouse CD16/32 antibody and Zombie NIR dye [31]. Each labeled monoclonal antibody including BV510conjugated anti-mouse Gr-1, PerCP-conjugated anti-mouse CD11b and APC-conjugated anti-mouse IL$18 \mathrm{R}$ was added into tubes before lysing erythrocytes. Finally, cells were processed as for human blood samples and analyzed using flow cytometry.

To detect IL-18R expression in NLF macrophages, cells were first incubated with anti-mouse CD16/32 antibodies and Zombie NIR dye, subsequently incubated with BV510-conjugated anti-mouse F4/80 and APC conjugated anti-mouse IL-18R antibodies, and analyzed as above.

\section{Real-time PCR for IL-18, IL-18BP and IL-18R in isolated CD14 ${ }^{+}$monocytes}

CDNA generated from total RNA of isolated human blood $\mathrm{CD} 14^{+}$cells was used as templates, and qPCR assay was performed as described by Zhang et al [32] using specific primers of IL-18, IL-18BP and IL-18R as listed in Supplement table 2. Each measurement of a sample was conducted in duplicate.

Measurement of cytokine levels in plasma and NLF supernatant, and calculation of molar concentration ratio of plasma IL-18BP/IL-18 
Levels of total IL-18 (tIL-18) and IL-18BP (tIL-18BP) in plasma or NLF supernatant were determined by using ELISA kit according to the manufacturer's instructions. Molar concentration of human IL-18 and IL18BP, and free IL-18 (fIL-18) and IL-18BP (fIL-18BP) were calculated as described by Novick D et al [33].

Human bio-plex panel (Bio-Rad Laboratories, California, USA) was employed to detect plasma levels of IL$1 \beta$ and TNF- $\alpha$. The detection ranges for IL-1 $\beta$ and TNF- $\alpha$ were $0.24-3994 \mathrm{pg} / \mathrm{mL}$ and $0.57-9270 \mathrm{pg} / \mathrm{mL}$, respectively.

\section{Statistical analysis}

Statistical analyses were performed by SPSS software (version 21.0, IBM Corporation). Data were displayed as a boxplot, which indicates the median, interquartile range, the largest and smallest values for indicated experiments. Where Kruskal-Wallis analysis indicated significant differences between groups, a pairwise test was used for multiple comparisons between the groups. Correlations were determined using Pearson's correlation or Spearman rank correlation analysis. For all analyses, $P<0.05$ was considered statistically significant.

\section{Abbreviations}

fIL-18

free IL-18

tIL-18

total IL-18

fIL-18BP

free IL-18BP

tIL-18BP

total IL-18BP

AR

allergic rhinitis

pAR

perennial allergic rhinitis

SAR

seasonal allergic rhinitis

$\mathrm{HC}$

healthy control subjects

$\mathrm{HM}$

healthy mice

PPAE

Platanus pollen allergen extract

DPAE

Dermatophagoides pteronyssinus allergen extract 
ASWAE

Artemisia sieversiana wild pollen allergen extrac

NLF

nasal lavage fluid

FMO

fluorescence minus one

MFI

mean fluorescence intensity

PBMC

peripheral blood mononuclear cells

\section{Declarations}

\section{Ethics approval and consent to participate}

The research was in compliance with the Declaration of Helsinki and was approved by the ethical committees of the First Affiliated Hospital of Jinzhou Medical University and the General Hospital of Northern Military Area. Animal ethics was approved by the Animal Care Committee at Jinzhou Medical University.

Written informed consent was obtained from all individual participants included in this study.

\section{Consent for publication}

Not applicable.

\section{Availability of data and materials}

The materials and data generated or analyzed during this study are included in this article and its supplementary information files. Raw data support the findings of this study will be available from the corresponding author upon reasonable request.

\section{Competing interests}

The authors declare they have no competing interest regarding the publication of this article.

All the authors including Junling Wang, Fangqiu Gu, Zhi Li, Mengmeng Zhan, Ling Wang, Huiyun Zhang, Dong Chen, Hua Xie, Ruonan Chai, Nan Zhao, Ruiming Yang, Yalin Hu and Shaoheng He have approved the manuscript and agree with submission to your journal.

\section{Funding}

This project was sponsored by the grants from the Key Laboratory of research on Pathogenesis of Allergen provoked Allergic Disease of Liaoning Province (2018-30); Allergic Disease Clinical Medicine 
Research Center of Liaoning Province (2017-37); Climbing Scholar Project for Institution of Higher Education in Liaoning province (2018-38), and the Natural Science Foundation of Liaoning Province (2019-ZD-0801; 201601354). All funders mentioned above provided funds only for reagent purchasing in this study, and had no role in study design, data collection and analysis, decision to publish, or preparation of the manuscript.

\section{Authors' contributions}

JL Wang and FQ Gu performed most experiments and drafted the original manuscript. MM Zhan, L Wang, RM Yang and YL Hu participated in magnetic cell sorting and flow cytometry. HY Zhang carried out ELISA and Bio-plex experiment, and generated the figures. D Chen, $\mathrm{H}$ Xie and RN Chai recruited volunteers and collected their general information. $\mathrm{N}$ Zhao carried out PCR experiment and data interpretation. Z Li contributed to animal study and literature review. SH He designed and supervised the study, analyzed the data and wrote the final version of the manuscript. All authors commented on previous manuscript, read and approved the final manuscript.

\section{Acknowledgments}

We would like to thank the nurses and allergists of the Allergy Clinic of the First Affiliated Hospital of Jinzhou Medical University in their daily work and efforts. We would also like to thank our volunteers for participating in this study.

\section{References}

1. Tungtrongchitr A, Jumpasri J, Sookrung N, Visitsunthorn N, Tantilipikorn P, Piboonpocanan O, et al Alteration of $-656(\mathrm{G} / \mathrm{T})$ and $-607(\mathrm{C} / \mathrm{A})$ polymorphisms in interleukin-18 (IL-18) gene in house dust mite-sensitive allergic rhinitis patients in Thailand. Genet Mol Res. 2017;16(3).

2. Lee HM, Park SA, Chung SW, Woo JS, Chae SW, Lee SH, et al. Interleukin-18/-607 gene polymorphism in allergic rhinitis. Int J Pediatr Otorhinolaryngol. 2006;70(6):1085-8.

3. Sebelova S, Izakovicova-Holla L, Stejskalova A, Schuller M, Znojil V, Vasku A. Interleukin-18 and its three gene polymorphisms relating to allergic rhinitis. J Hum Genet. 2007;52(2):152-8.

4. Ibrahim GH, ElTabbakh MT, Gomaa AH, Mohamed EA. Interleukin-18 gene polymorphisms in Egyptian patients with allergic diseases. Am J Rhinol Allergy. 2012;26(5):385-9.

5. Kruse S, Kuehr J, Moseler M, Kopp MV, Kurz T, Deichmann KA, et al. Polymorphisms in the IL 18 gene are associated with specific sensitization to common allergens and allergic rhinitis. J Allergy Clin Immunol. 2003;111(1):117-22.

6. Verhaeghe B, Gevaert P, Holtappels G, Lukat KF, Lange B, Van Cauwenberge P, et al. Up-regulation of IL-18 in allergic rhinitis. Allergy. 2002;57(9):825-30.

7. Ando M, Shima M. Serum interleukins 12 and 18 and immunoglobulin E concentrations and allergic symptoms in Japanese schoolchildren. J Investig Allergol Clin Immunol. 2007;17(1):14-9. 
8. Kurt E, Aksu K, Dokumacioglu A, Alatas O. Bronchial hyperresponsiveness in seasonal allergic rhinitis patients is associated with increased IL-18 during natural pollen exposure. Cytokine. 2012;60(1):100-3.

9. Adachi O, Kawai T, Takeda K, Matsumoto M, Tsutsui H, Sakagami M, et al. Targeted disruption of the MyD88 gene results in loss of IL-1- and IL-18-mediated function. Immunity. 1998;9(1):143-50.

10. Novick D, Kim SH, Fantuzzi G, Reznikov LL, Dinarello CA, Rubinstein M. Interleukin-18 binding protein: a novel modulator of the Th1 cytokine response. Immunity. 1999;10(1):127-36.

11. Girard C, Rech J, Brown M, Allali D, Roux-Lombard P, Spertini F, et al. Elevated serum levels of free interleukin-18 in adult-onset Still's disease. Rheumatology. 2016;55(12):2237-47.

12. Hu Y, Wang J, Zhang H, Xie H, Song W, Jiang Q, et al. Enhanced Expression of IL-18 and IL-18BP in Plasma of Patients with Eczema: Altered Expression of IL-18BP and IL-18 Receptor on Mast Cells. Mediators Inflamm. 2017;2017:3090782.

13. Zhang $\mathrm{H}$, Wang J, Wang $\mathrm{L}$, Xie $H$, Chen $L$, He S. Role of IL-18 in atopic asthma is determined by balance of IL-18/IL-18BP/IL-18R. J Cell Mol Med. 2018;22(1):354-73.

14. Ariano R, Merendino RA, Minciullo PL, Salpietro CD, Gangemi S. Specific immunotherapy effect on interleukin-18 and CD30 serum levels in monosensitized patients with rhinitis. Allergy Asthma Proc. 2003;24(3):179 - 83.

15. Nieminen K, Laaksonen K, Savolainen J. Three-year follow-up study of allergen-induced in vitro cytokine and signalling lymphocytic activation molecule mRNA responses in peripheral blood mononuclear cells of allergic rhinitis patients undergoing specific immunotherapy. Int Arch Allergy Immunol. 2009;150(4):370-6.

16. Savolainen J, Nieminen K, Laaksonen K, Laiho T, Jacobsen L, Lahesmaa R, et al. Allergen-induced in vitro expression of IL-18, SLAM and GATA-3 mRNA in PBMC during sublingual immunotherapy. Allergy. 2007;62(8):949-53.

17. Savolainen J, Laaksonen K, Rantio-Lehtimaki A, Terho EO. Increased expression of allergen-induced in vitro interleukin-10 and interleukin-18 mRNA in peripheral blood mononuclear cells of allergic rhinitis patients after specific immunotherapy. Clin Exp Allergy. 2004;34(3):413-9.

18. Kahlenberg JM, Dubyak GR. Differing caspase-1 activation states in monocyte versus macrophage models of IL-1beta processing and release. J Leukoc Biol. 2004;76(3):676-84.

19. Shi L, Yin W, Zhang Z, Shi G. Intestinal dysbacteriosis potentiates ovalbumin-induced allergic airway inflammation by inhibiting microRNA-130a to upregulate tumor necrosis factor alpha. Int Immunopharmacol. 2018;60:34-40.

20. Shi Q, Lei Z, Cheng G, Li D, Wang Q, Luo S, et al. Mitochondrial ROS activate interleukin-1beta expression in allergic rhinitis. Oncol Lett. 2018;16(3):3193-200.

21. Eguiluz-Gracia I, Bosco A, Dollner R, Melum GR, Lexberg MH, Jones AC, et al. Rapid recruitment of CD14(+) monocytes in experimentally induced allergic rhinitis in human subjects. J Allergy Clin Immunol. 2016;137(6):1872-81. e12. 
22. Sanders NL, Mishra A. Role of interleukin-18 in the pathophysiology of allergic diseases. Cytokine Growth Factor Rev. 2016;32:31-9.

23. Leach ST, Messina I, Lemberg DA, Novick D, Rubenstein M, Day AS. Local and systemic interleukin-18 and interleukin-18-binding protein in children with inflammatory bowel disease. Inflamm Bowel Dis. 2008;14(1):68-74.

24. Piccioli P, Rubartelli A. The secretion of IL-1beta and options for release. Semin Immunol. 2013;25(6):425-9.

25. Aggarwal BB, Gupta SC, Kim JH. Historical perspectives on tumor necrosis factor and its superfamily: 25 years later, a golden journey. Blood. 2012;119(3):651-65.

26. Kaplanski G. Interleukin-18: Biological properties and role in disease pathogenesis. Immunol Rev. 2018;281(1):138-53.

27. Kandikattu HK, Upparahalli Venkateshaiah S, Mishra A. Synergy of Interleukin (IL)-5 and IL-18 in eosinophil mediated pathogenesis of allergic diseases. Cytokine Growth Factor Rev. 2019;47:83-98.

28. Cheng L, Chen J, Fu Q, He S, Li H, Liu Z, et al. Chinese Society of Allergy Guidelines for Diagnosis and Treatment of Allergic Rhinitis. Allergy Asthma Immunol Res. 2018;10(4):300-53.

29. Freemerman AJ, Zhao L, Pingili AK, Teng B, Cozzo AJ, Fuller AM, et al. Myeloid Slc2a1-Deficient Murine Model Revealed Macrophage Activation and Metabolic Phenotype Are Fueled by GLUT1. J Immunol. 2019;202(4):1265-86.

30. Mo JH, Kang EK, Quan SH, Rhee CS, Lee CH, Kim DY. Anti-tumor necrosis factor-alpha treatment reduces allergic responses in an allergic rhinitis mouse model. Allergy. 2011;66(2):279-86.

31. Headley MB, Bins A, Nip A, Roberts EW, Looney MR, Gerard A, et al. Visualization of immediate immune responses to pioneer metastatic cells in the lung. Nature. 2016;531(7595):513-7.

32. Zhang $H$, Yang $X$, Yang $H$, Zhang $Z$, Lin $Q$, Zheng $Y$, et al. Modulation of mast cell proteinaseactivated receptor expression and IL-4 release by IL-12. Immunol Cell Biol. 2007;85(7):558-66.

33. Novick D, Schwartsburd B, Pinkus R, Suissa D, Belzer I, Sthoeger Z, et al. A novel IL-18BP ELISA shows elevated serum IL-18BP in sepsis and extensive decrease of free IL-18. Cytokine. 2001;14(6):334-42.

\section{Figures}



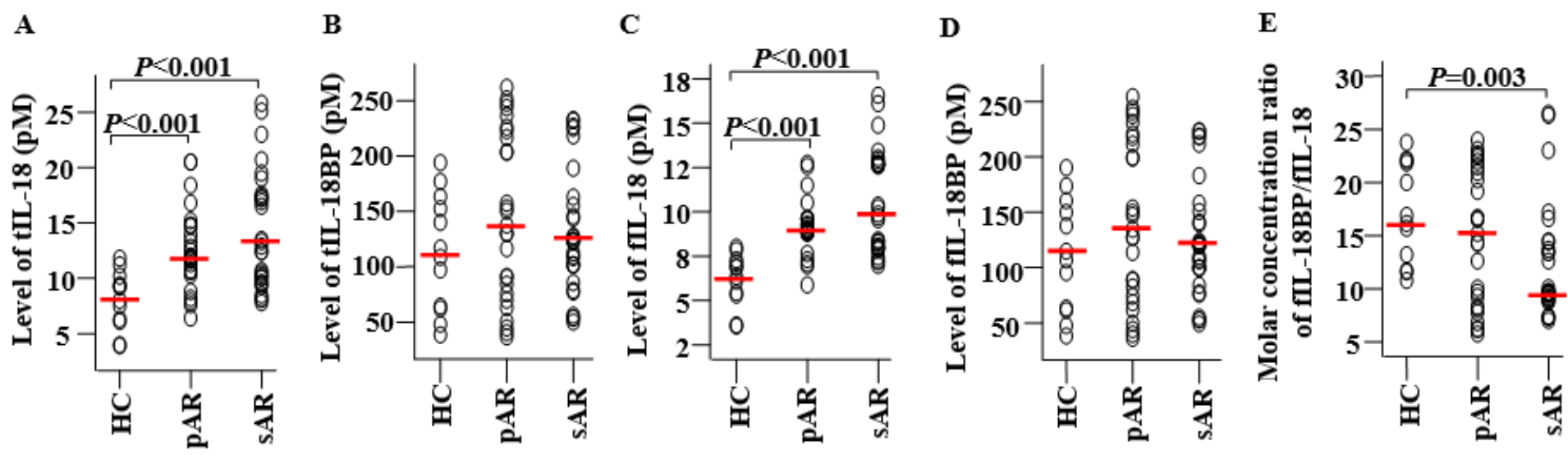

F Pearson's correlation coefficients between plasma fIL-18 and fIL-18BP in AR patients and $\mathrm{HC}$

\begin{tabular}{|c|c|c|c|c|c|c|}
\hline \multirow{3}{*}{ Compound } & \multicolumn{6}{|c|}{ Correlation between plasma fIL-18 and fIL-18BP ( $r$ values) } \\
\hline & \multicolumn{2}{|c|}{$\mathrm{pAR}$} & \multicolumn{2}{|c|}{ SAR } & \multicolumn{2}{|c|}{$\mathrm{HC}$} \\
\hline & fIL-18 & fIL-18BP & fIL-18 & fIL-18BP & fIL-18 & fIL-18BP \\
\hline fIL-18 & 1 & $0.920^{*}$ & 1 & $0.979^{*}$ & 1 & $0.993^{*}$ \\
\hline fIL-18BP & $0.920 *$ & 1 & $0.979 *$ & 1 & $0.993 *$ & 1 \\
\hline
\end{tabular}

\section{Figure 1}

Scatter plots of levels of total IL-18 (tIL-18, A) and total IL-18BP (tIL-18, B), free IL-18 (fIL-18, C) and free IL-18BP (fIL-18, D) in plasma of patients with perennial allergic rhinitis (pAR) and seasonal allergic rhinitis (SAR), and healthy control subjects (HC). (E) shows the molar concentration ratios of fIL-18BP/fIL18. Each symbol represents the value from one subject. The median value of each defined group of subjects is indicated as a horizontal line. The Pearson's correlation coefficient between plasma levels of fIL-18 and fIL-18BP is shown in (F). P $<0.05$ was taken as statistically significant. 

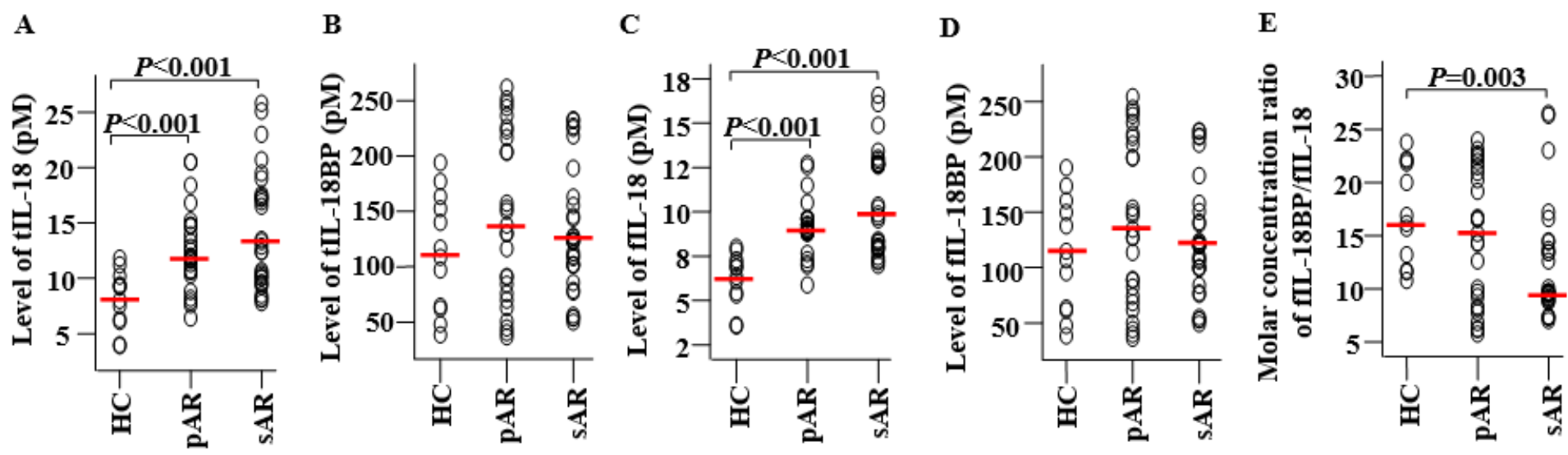

F Pearson's correlation coefficients between plasma fIL-18 and fIL-18BP in AR patients and $\mathrm{HC}$

\begin{tabular}{|c|c|c|c|c|c|c|}
\hline \multirow{3}{*}{ Compound } & \multicolumn{6}{|c|}{ Correlation between plasma fIL-18 and fIL-18BP ( $r$ values) } \\
\hline & \multicolumn{2}{|c|}{$\mathrm{pAR}$} & \multicolumn{2}{|c|}{ SAR } & \multicolumn{2}{|c|}{$\mathrm{HC}$} \\
\hline & fIL-18 & fIL-18BP & fIL-18 & fIL-18BP & fIL-18 & fIL-18BP \\
\hline fIL-18 & 1 & $0.920^{*}$ & 1 & $0.979^{*}$ & 1 & $0.993^{*}$ \\
\hline fIL-18BP & $0.920 *$ & 1 & $0.979 *$ & 1 & $0.993 *$ & 1 \\
\hline
\end{tabular}

\section{Figure 1}

Scatter plots of levels of total IL-18 (tIL-18, A) and total IL-18BP (tIL-18, B), free IL-18 (fIL-18, C) and free IL-18BP (fIL-18, D) in plasma of patients with perennial allergic rhinitis (pAR) and seasonal allergic rhinitis (SAR), and healthy control subjects (HC). (E) shows the molar concentration ratios of fIL-18BP/fIL18. Each symbol represents the value from one subject. The median value of each defined group of subjects is indicated as a horizontal line. The Pearson's correlation coefficient between plasma levels of fIL-18 and fIL-18BP is shown in (F). P $<0.05$ was taken as statistically significant. 
A

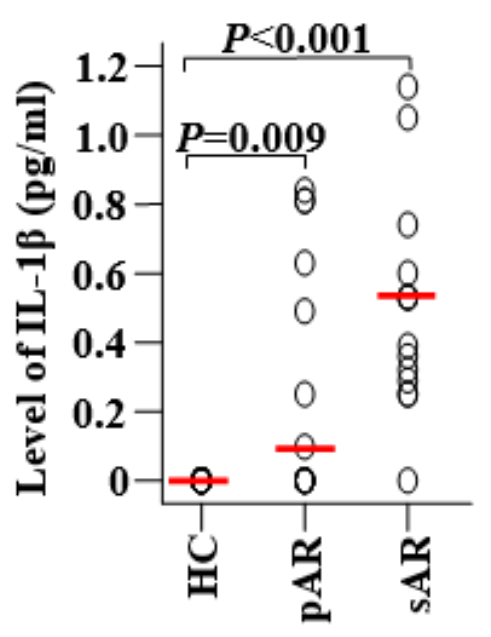

B

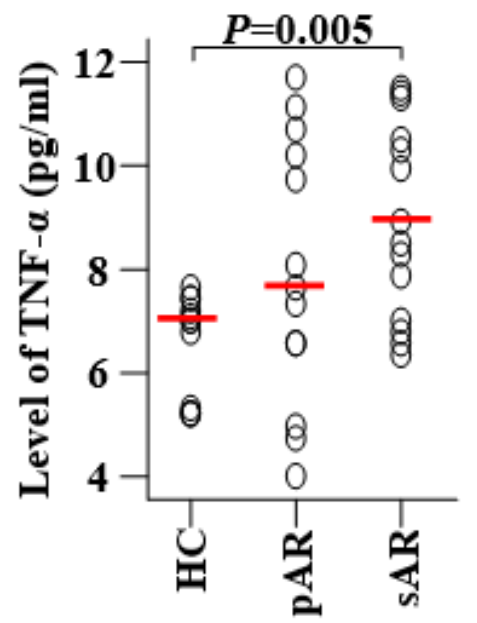

C Rank correlation (Spearman's $\rho$ correlation coefficient) among plasma fIL-18, IL-1 $\beta$ and TNF- $\alpha$ in $\mathrm{pAR}$ and SAR patients.

\begin{tabular}{lccccccc}
\hline \multirow{5}{*}{ Compound } & \multicolumn{3}{c}{ pAR } & & & \multicolumn{3}{c}{ sAR } \\
\cline { 2 - 6 } \cline { 6 - 8 } & fIL-18 & IL-1 $\beta$ & TNF- $\alpha$ & & flL-18 & IL-1 $\beta$ & TNF- $\alpha$ \\
\hline fIL-18 & 1 & $0.865^{*}$ & $0.965^{*}$ & & 1 & $0.975^{*}$ & $0.998^{*}$ \\
IL-1 $\beta$ & $0.865^{*}$ & 1 & $0.921^{*}$ & & $0.975^{*}$ & 1 & $0.985^{*}$ \\
TNF- $\alpha$ & $0.965^{*}$ & $0.921^{*}$ & 1 & & $0.998^{*}$ & $0.985^{*}$ & 1 \\
\hline
\end{tabular}

$* P<0.05$.

Figure 2

Scatter plots of levels of IL-1 $\beta(A)$ and TNF- $\alpha(B)$ in plasma of perennial allergic rhinitis (pAR) and seasonal allergic rhinitis (SAR) patients and healthy control subjects $(\mathrm{HC})$. Each symbol represents the value from one subject. The median value of each defined group of subjects is indicated as a horizontal line. The Spearman's $\rho$ correlation coefficient between the plasma levels of $f I L-18, I L-1 \beta$ and TNF- $\alpha$ in $p A R$ and SAR patients is shown in (C). $P<0.05$ was taken as statistically significant. 
A

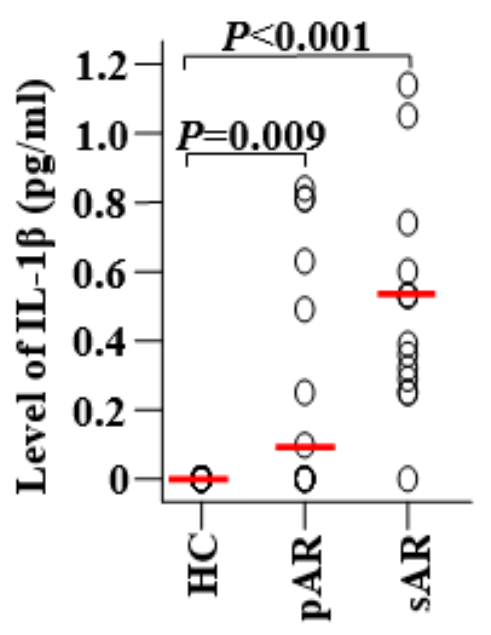

B

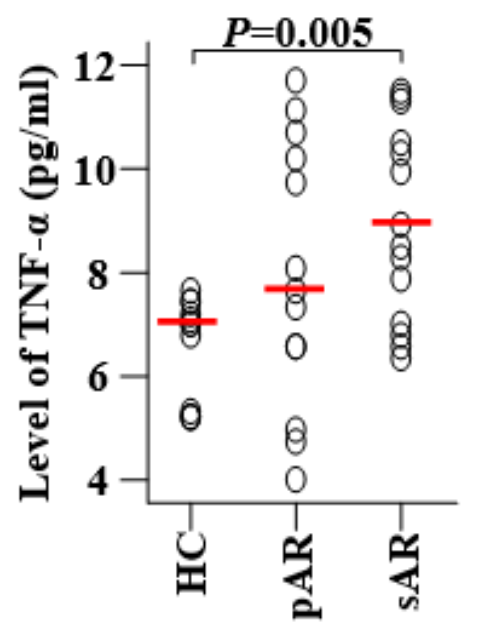

C Rank correlation (Spearman's $\rho$ correlation coefficient) among plasma fIL-18, IL-1 $\beta$ and TNF- $\alpha$ in $\mathrm{pAR}$ and SAR patients.

\begin{tabular}{lccccccc}
\hline \multirow{5}{*}{ Compound } & \multicolumn{3}{c}{ pAR } & & & \multicolumn{3}{c}{ sAR } \\
\cline { 2 - 6 } \cline { 6 - 8 } & fIL-18 & IL-1 $\beta$ & TNF- $\alpha$ & & fIL-18 & IL-1 $\beta$ & TNF- $\alpha$ \\
\hline fIL-18 & 1 & $0.865^{*}$ & $0.965^{*}$ & & 1 & $0.975^{*}$ & $0.998^{*}$ \\
IL-1 $\beta$ & $0.865^{*}$ & 1 & $0.921^{*}$ & & $0.975^{*}$ & 1 & $0.985^{*}$ \\
TNF- $\alpha$ & $0.965^{*}$ & $0.921^{*}$ & 1 & & $0.998^{*}$ & $0.985^{*}$ & 1 \\
\hline
\end{tabular}

$* P<0.05$.

Figure 2

Scatter plots of levels of IL-1 $\beta(A)$ and TNF- $\alpha(B)$ in plasma of perennial allergic rhinitis (pAR) and seasonal allergic rhinitis (SAR) patients and healthy control subjects $(\mathrm{HC})$. Each symbol represents the value from one subject. The median value of each defined group of subjects is indicated as a horizontal line. The Spearman's $\rho$ correlation coefficient between the plasma levels of $\mathrm{fIL}-18, \mathrm{IL}-1 \beta$ and TNF- $\alpha$ in $p A R$ and SAR patients is shown in (C). $P<0.05$ was taken as statistically significant. 

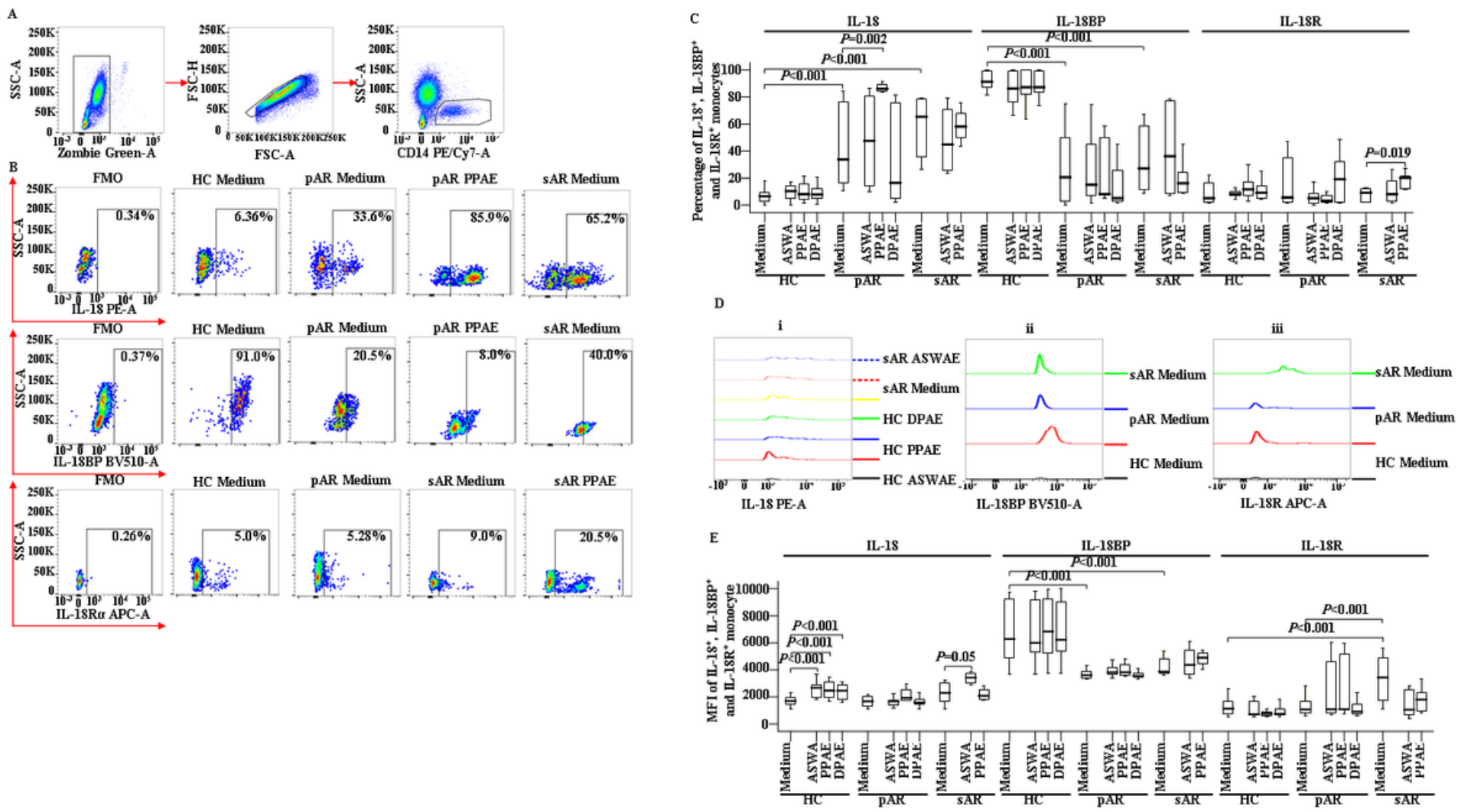

\section{Figure 3}

Expression of IL-18, IL-18BP and IL-18R in peripheral blood CD14+ monocytes of patients with perennial allergic rhinitis (pAR) and seasonal allergic rhinitis (SAR), and healthy control subjects $(H C)$ in the presence or absence of Dermatophagoides pteronyssinus allergen extract (DPAE), Artemisia sieversiana wild allergen extract (ASWAE) and Platanus pollen allergen extract (PPAE). (A) represents a gating strategy of CD14+ monocytes in leukocytes; $(B)$ is representitive figures of proportions of IL-18+, IL-18BP+ and IL-18R+ cells in CD14+ monocytes; (C) demonstrates percentages of IL-18, IL-18BP and IL-18R expressing monocytes in CD14+ monocytes; (D) shows representative flow cytometric figures of mean fluorescence intensity (MFI) of IL-18+ (i), IL-18BP+ (ii) and IL-18R+ (iii) CD14+ monocyte. (E) reveals MFI levels of IL-18, IL-18BP and IL-18R expression in CD14+ monocyte. Data are displayed as a boxplot for pAR patients $(n=33)$, sAR patients $(n=9)$, and $H C(n=25)$, which indicates the median, interquartile range, the largest and smallest values for the number of volunteers indicated. $\mathrm{P}<0.05$ was taken as statistically significant. FMO = fluorescence minus one control. 

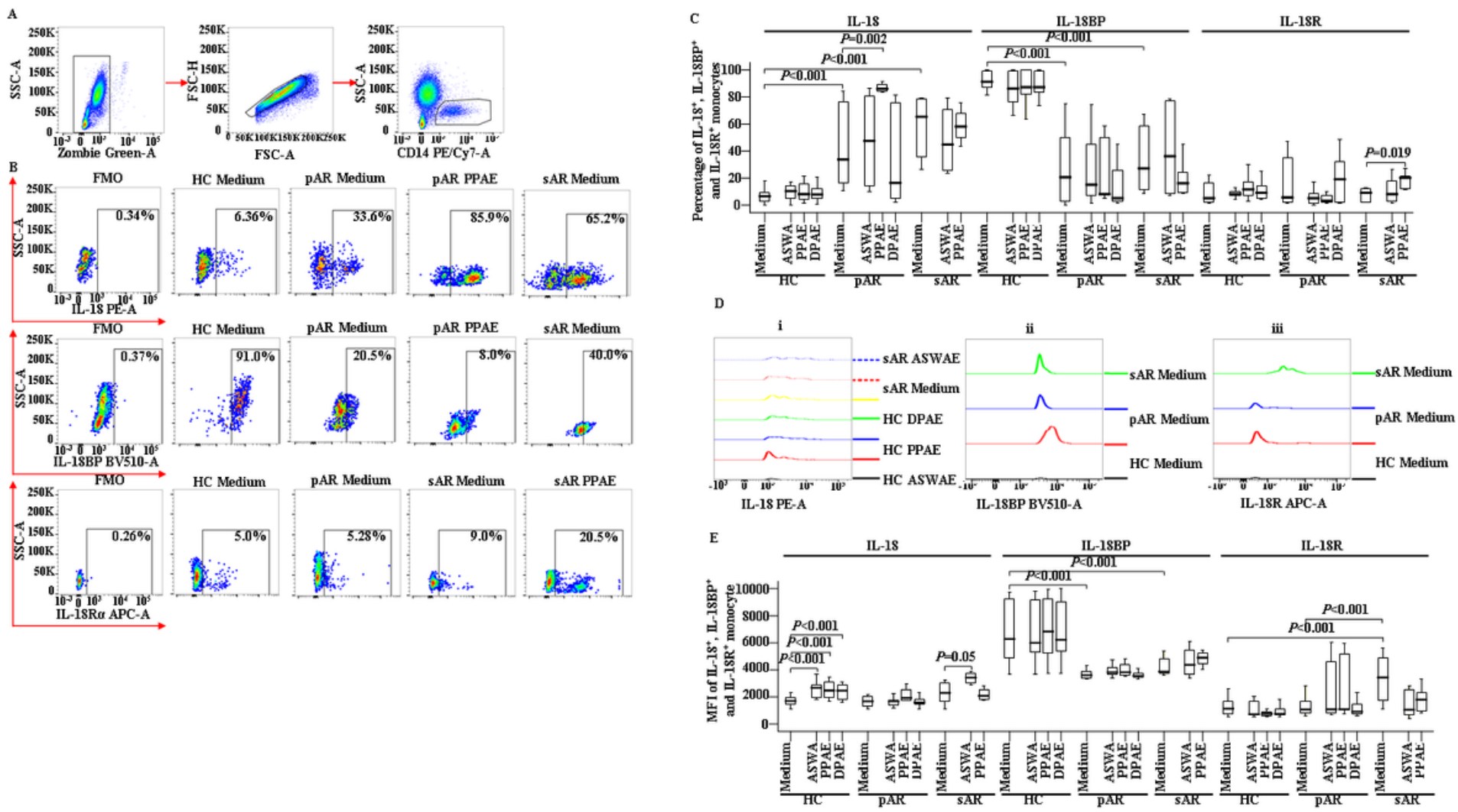

\section{Figure 3}

Expression of IL-18, IL-18BP and IL-18R in peripheral blood CD14+ monocytes of patients with perennial allergic rhinitis (pAR) and seasonal allergic rhinitis (SAR), and healthy control subjects $(H C)$ in the presence or absence of Dermatophagoides pteronyssinus allergen extract (DPAE), Artemisia sieversiana wild allergen extract (ASWAE) and Platanus pollen allergen extract (PPAE). (A) represents a gating strategy of CD14+ monocytes in leukocytes; $(B)$ is representitive figures of proportions of IL-18+, IL-18BP+ and IL-18R+ cells in CD14+ monocytes; (C) demonstrates percentages of IL-18, IL-18BP and IL-18R expressing monocytes in CD14+ monocytes; (D) shows representative flow cytometric figures of mean fluorescence intensity (MFI) of IL-18+ (i), IL-18BP+ (ii) and IL-18R+ (iii) CD14+ monocyte. (E) reveals MFI levels of IL-18, IL-18BP and IL-18R expression in CD14+ monocyte. Data are displayed as a boxplot for pAR patients $(n=33)$, sAR patients $(n=9)$, and $H C(n=25)$, which indicates the median, interquartile range, the largest and smallest values for the number of volunteers indicated. $\mathrm{P}<0.05$ was taken as statistically significant. FMO = fluorescence minus one control. 

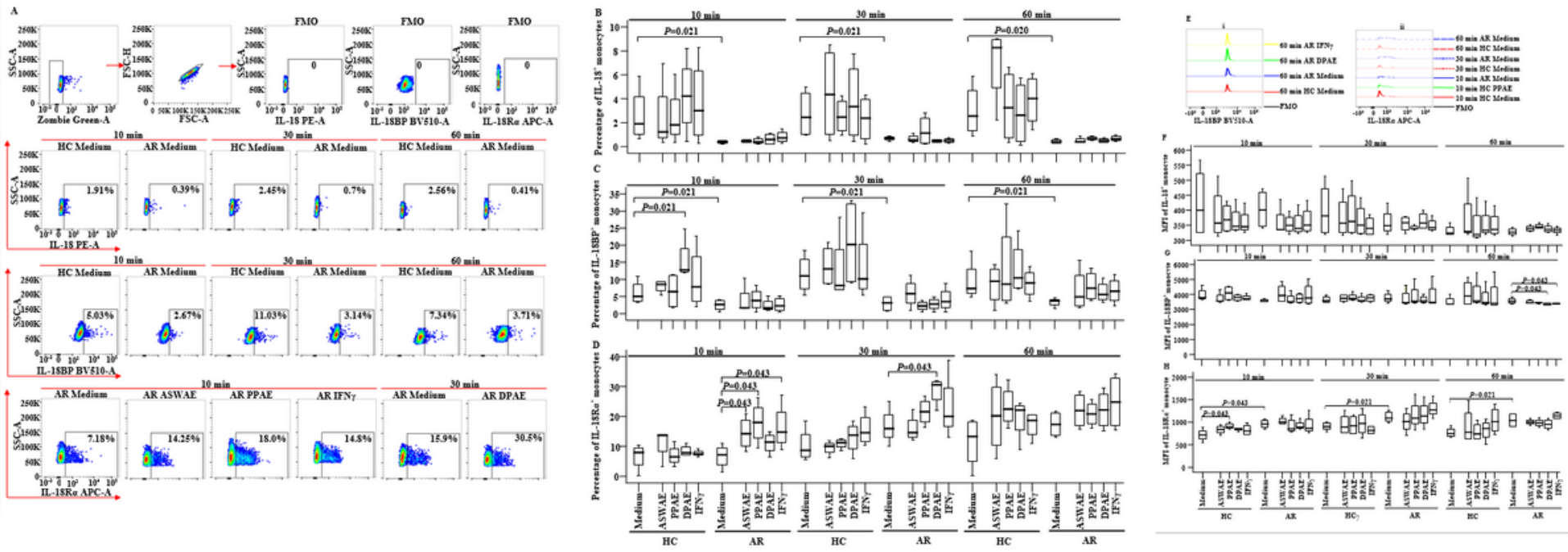

Figure 4

Expression of IL-18, IL-18BP and IL-18R in isolated monocytes of allergic rhinitis (AR) patients and healthy control subjects $(\mathrm{HC})$ in the presence or absence of Dermatophagoides pteronyssinus allergen extract (DPAE), Artemisia sieversiana wild allergen extract (ASWAE) and Platanus pollen allergen extract (PPAE). (A) is a gating strategy of expression of IL-18, IL-18BP and IL-18R in isolated monocytes; (B, C, D) demonstrate percentages of IL-18, IL-18BP and IL-18R expressing monocytes, respectively; (E) shows representative figures of mean fluorescence intensity (MFI) of IL-18BP+ (i) and IL-18R+ (ii) monocyte. (F, $\mathrm{G}, \mathrm{H})$ reveal MFI of IL-18, IL-18BP and IL-18R expression in monocyte, respectively. Data are displayed as a boxplot for AR patients $(n=6)$ and $\mathrm{HC}(n=6)$, which indicates the median, interquartile range, the largest and smallest values for the number of volunteers indicated. FMO = fluorescence minus one control. $\mathrm{P}<0.05$ was taken as statistically significant.
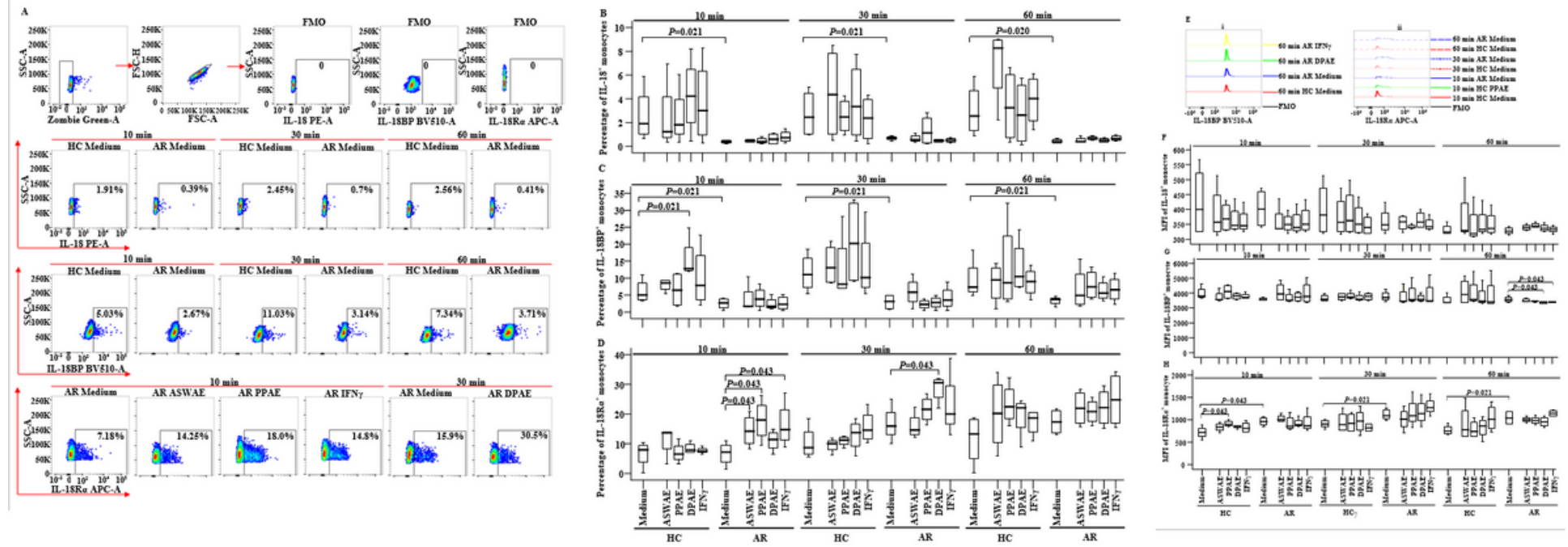

Figure 4

Expression of IL-18, IL-18BP and IL-18R in isolated monocytes of allergic rhinitis (AR) patients and healthy control subjects $(\mathrm{HC})$ in the presence or absence of Dermatophagoides pteronyssinus allergen 
extract (DPAE), Artemisia sieversiana wild allergen extract (ASWAE) and Platanus pollen allergen extract (PPAE). (A) is a gating strategy of expression of IL-18, IL-18BP and IL-18R in isolated monocytes; $(B, C, D)$ demonstrate percentages of IL-18, IL-18BP and IL-18R expressing monocytes, respectively; (E) shows representative figures of mean fluorescence intensity (MFI) of IL-18BP+ (i) and IL-18R+ (ii) monocyte. (F, G, H) reveal MFI of IL-18, IL-18BP and IL-18R expression in monocyte, respectively. Data are displayed as a boxplot for AR patients $(n=6)$ and $H C(n=6)$, which indicates the median, interquartile range, the largest and smallest values for the number of volunteers indicated. $\mathrm{FMO}=$ fluorescence minus one control. $\mathrm{P}<0.05$ was taken as statistically significant.

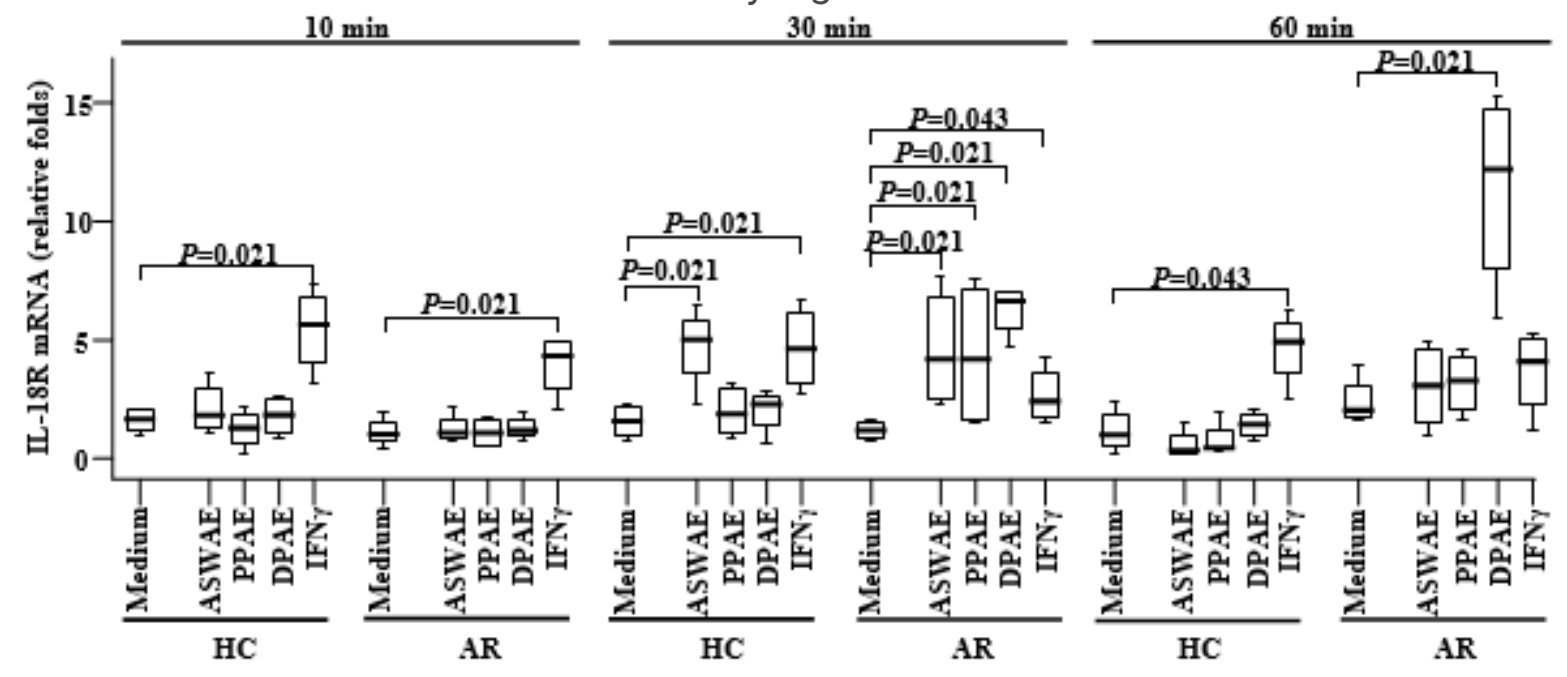

\section{Figure 5}

Quantitative real-time PCR (qPCR) analysis of expression of IL-18 receptor (IL-18R) mRNA in isolated monocytes of patients with allergic rhinitis (AR) and healthy control subjects (HC). Cells were incubated in the presence or absence of Artemisia sieversiana wild allergen extract (ASWAE), Platanus pollen allergen extract (PPAE) and Dermatophagoides allergen extract (DPAE) or IFNy. Expression of IL-18R mRNA was analyzed by qPCR. The data displayed as a boxplot for AR patients $(n=6)$ and HC $(n=6)$, which indicates the median, interquartile range, the largest and smallest values for the number of volunteers indicated. $\mathrm{P}<0.05$ was taken as statistically significant.

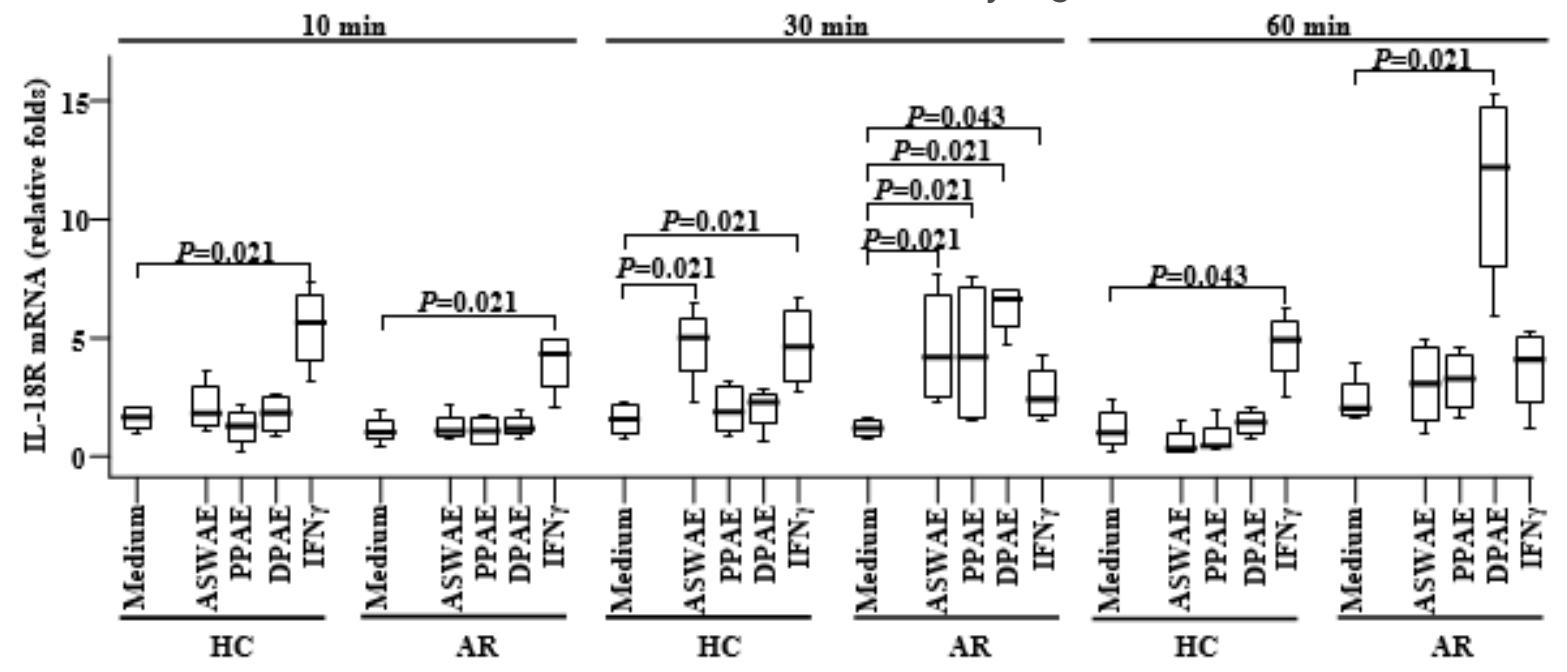




\section{Figure 5}

Quantitative real-time PCR (qPCR) analysis of expression of IL-18 receptor (IL-18R) mRNA in isolated monocytes of patients with allergic rhinitis (AR) and healthy control subjects (HC). Cells were incubated in the presence or absence of Artemisia sieversiana wild allergen extract (ASWAE), Platanus pollen allergen extract (PPAE) and Dermatophagoides allergen extract (DPAE) or IFNy. Expression of IL-18R mRNA was analyzed by qPCR. The data displayed as a boxplot for AR patients $(n=6)$ and $H C(n=6)$, which indicates the median, interquartile range, the largest and smallest values for the number of volunteers indicated. $\mathrm{P}<0.05$ was taken as statistically significant.

A

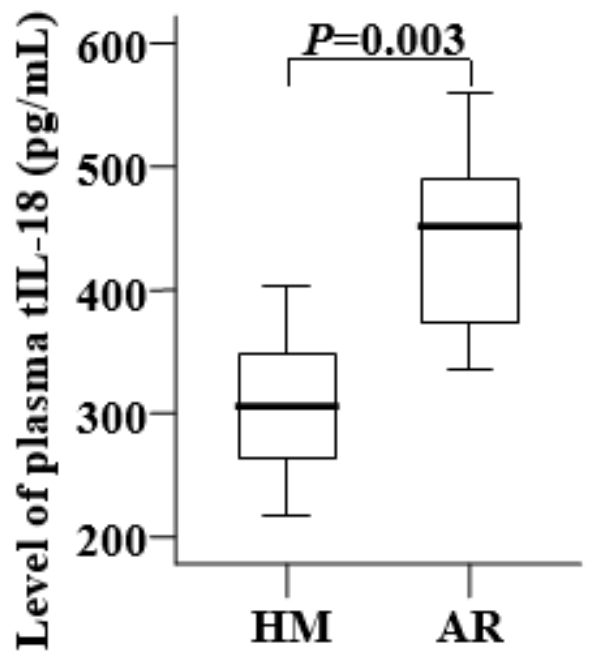

B

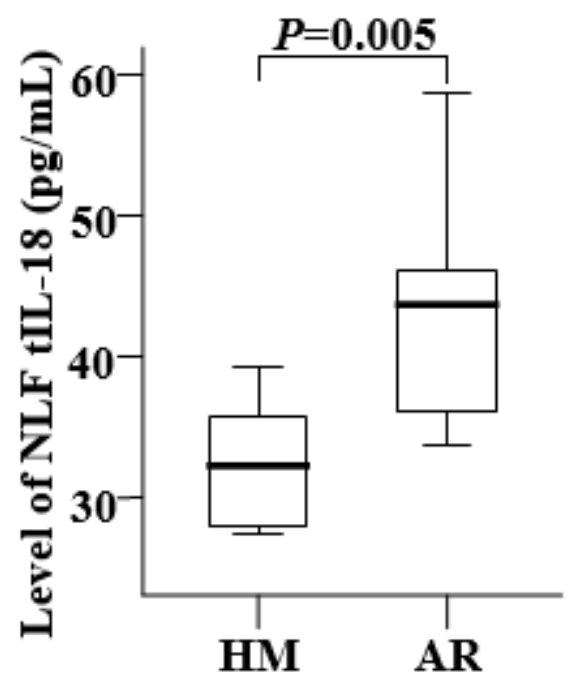

Figure 6

Levels of IL-18 in mouse plasma (A) and NLF (B). Following a seven-day OVA challergen or vehicle control treatment, plasma and NLF supernatant of OVA-induced allergic rhinitis (AR) mice and healthy mice (HM) were taken, and analyzed by using sandwich ELISA kits. Data are displayed as a boxplot for AR mice $(n=7)$ and HM $(n=7)$, which indicates the median, interquartile range, the largest and smallest values for the number of animals indicated. $P<0.05$ was taken as significant. 
$\mathbf{A}$

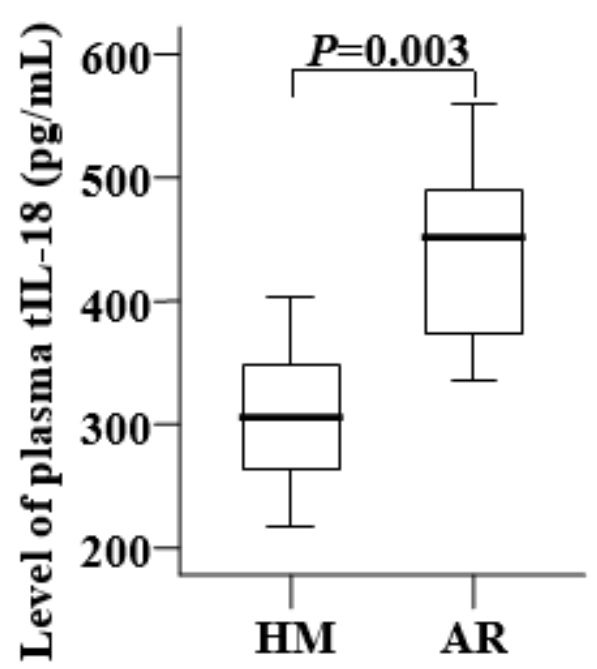

B

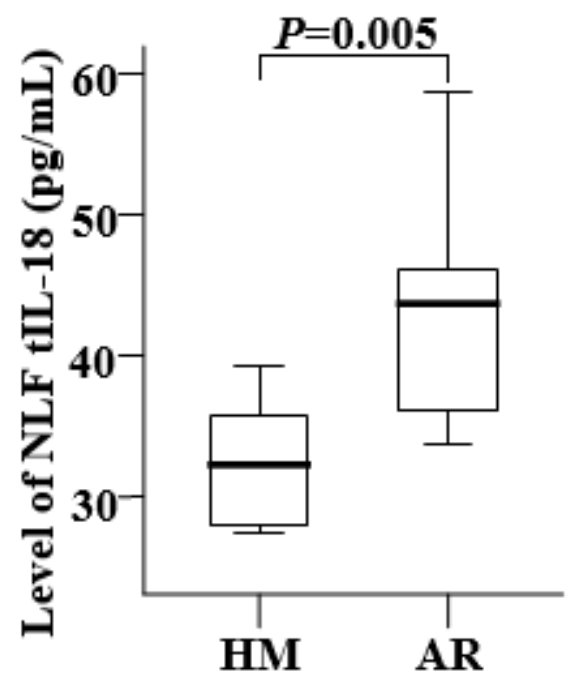

Figure 6

Levels of IL-18 in mouse plasma (A) and NLF (B). Following a seven-day OVA challergen or vehicle control treatment, plasma and NLF supernatant of OVA-induced allergic rhinitis (AR) mice and healthy mice (HM) were taken, and analyzed by using sandwich ELISA kits. Data are displayed as a boxplot for AR mice $(n=7)$ and HM $(n=7)$, which indicates the median, interquartile range, the largest and smallest values for the number of animals indicated. $\mathrm{P}<0.05$ was taken as significant.
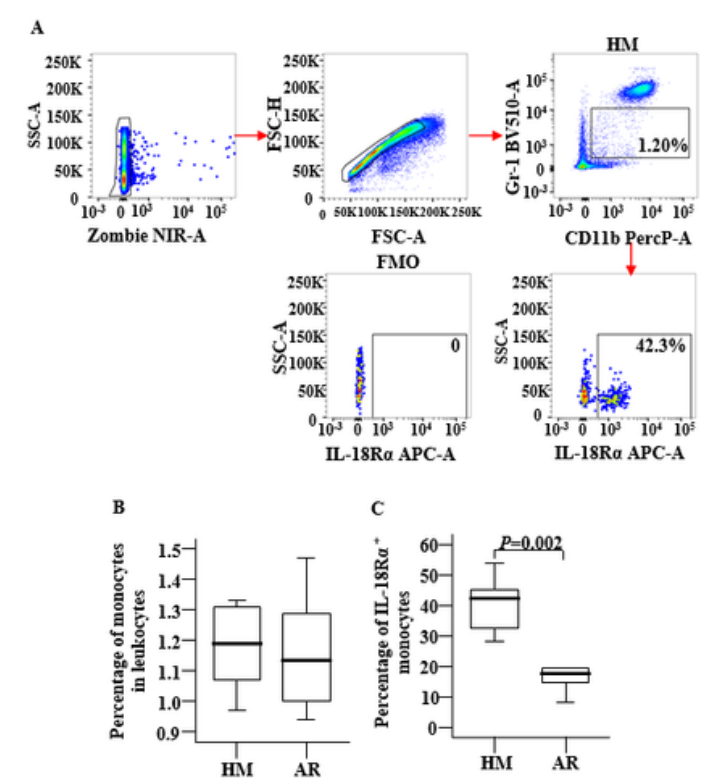

$\mathrm{C}$

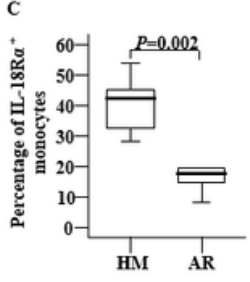

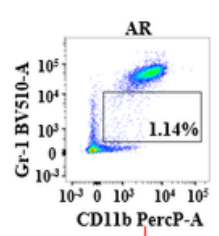

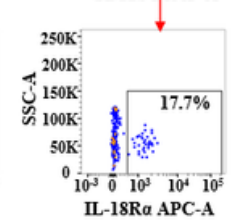

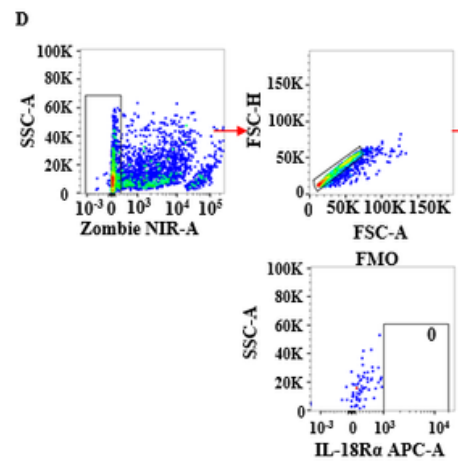
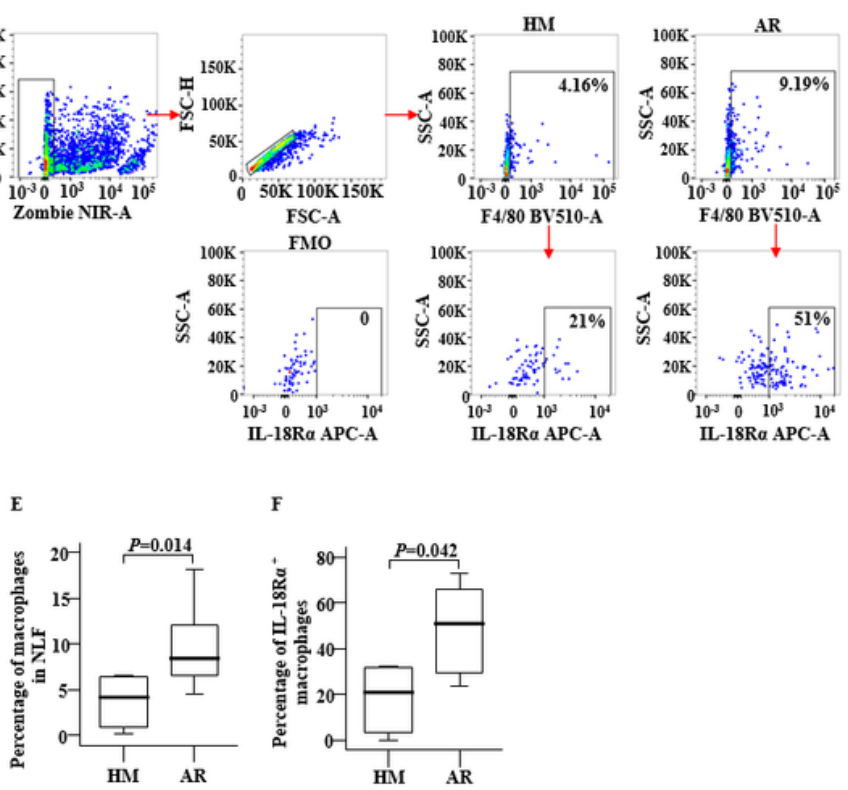

\section{Figure 7}

Expression of IL-18 receptor (R) in blood monocytes and nasal lavage fluid (NLF) macrophages of OVAinduced allergic rhinitis (AR) mice or vehicle treated healthy mice $(\mathrm{HM})$. (A) shows a gating strategy of CD11b+ Gr-1low monocyte expression in mouse leukocytes, and IL-18R expression in monocytes; (B, C) 
demonstrate percentages of monocytes in leukocytes, and proportions of IL-18R expressing monocytes, respectively; (D) represents a gating strategy of F4/80+ macrophage expression in mouse NLF, and IL$18 R$ expression in macrophages of mouse NLF. (E, F) reveal percentages of macrophages in NLF, and proportions of IL-18R expressing NLF macrophages, respectively. Data are displayed as a boxplot for AR mice $(n=7)$ and $\mathrm{HM}(n=7)$, which indicates the median, interquartile range, the largest and smallest values for the number of subjects indicated. $\mathrm{P}<0.05$ was taken as statistically significant. $F M O=$ fluorescence minus one control.
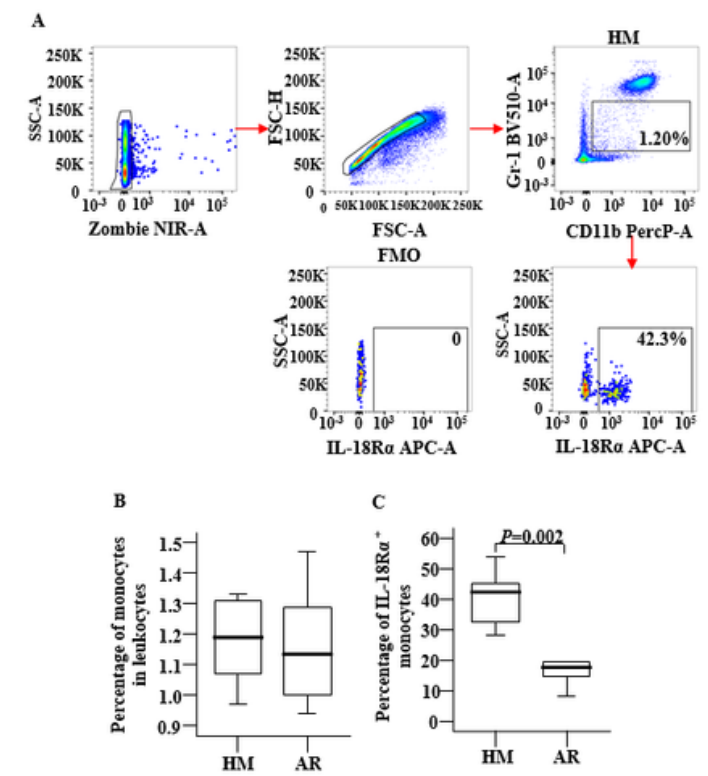
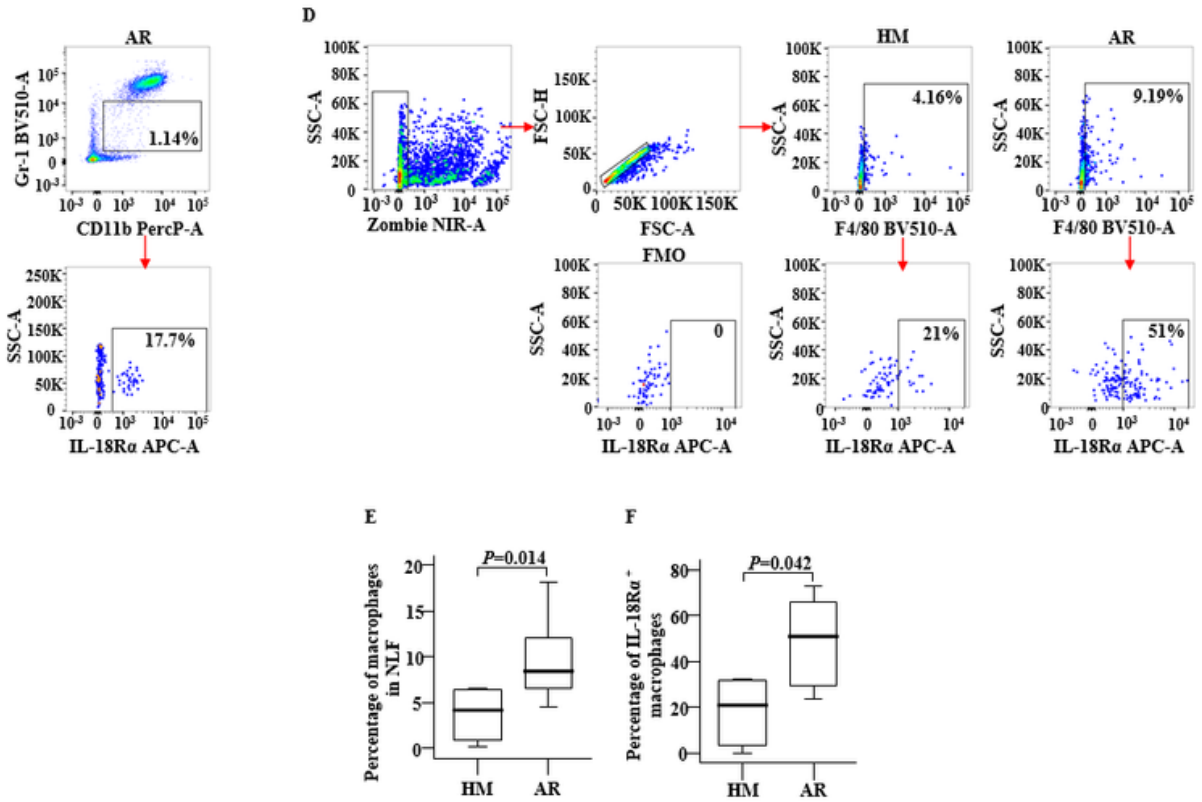

Figure 7

Expression of IL-18 receptor (R) in blood monocytes and nasal lavage fluid (NLF) macrophages of OVAinduced allergic rhinitis (AR) mice or vehicle treated healthy mice (HM). (A) shows a gating strategy of $\mathrm{CD} 11 \mathrm{~b}+\mathrm{Gr}-1$ low monocyte expression in mouse leukocytes, and IL-18R expression in monocytes; $(\mathrm{B}, \mathrm{C})$ demonstrate percentages of monocytes in leukocytes, and proportions of IL-18R expressing monocytes, respectively; (D) represents a gating strategy of F4/80+ macrophage expression in mouse NLF, and IL$18 \mathrm{R}$ expression in macrophages of mouse NLF. $(E, F)$ reveal percentages of macrophages in NLF, and proportions of IL-18R expressing NLF macrophages, respectively. Data are displayed as a boxplot for AR mice $(n=7)$ and $\mathrm{HM}(n=7)$, which indicates the median, interquartile range, the largest and smallest values for the number of subjects indicated. $\mathrm{P}<0.05$ was taken as statistically significant. $\mathrm{FMO}=$ fluorescence minus one control.

\section{Supplementary Files}

This is a list of supplementary files associated with this preprint. Click to download.

- ARRIVEchecklist.docx

- ARRIVEchecklist.docx 
- SupplementalMaterialIL18IL18RinMonocytesofAR.doc

- SupplementalMaterialIL18IL18RinMonocytesofAR.doc 